\title{
GÅRDING INEQUALITY FOR HIGHER ORDER ELLIPTIC OPERATORS WITH A NON-POWER DEGENERATION AND ITS APPLICATIONS
}

\author{
S.A. ISKHOKOV, M.G. GADOEV, I.YA. YAKUSHEV
}

\begin{abstract}
For higher order elliptic operators in an arbitrary (bounded or unbouned) domain in $n$-dimensional Euclidean space $\mathbb{R}_{n}$ with a non-power degeneration we prove a weighted analogue of Carding inequality. By means of this inequality we study the unique solvability of the Dirichlet variational problem, whose solution is sought in the closure of the class of infinitely differentiable compactly supported functions. The degeneration of the coefficients in different variables is characterized via different functions. The lower coefficients of the operators are assumed to belong to some weighted $L_{p}$-spaces. For one class of elliptic operators with a power degeneration in a half-space we study the solvability of variational Dirichlet problem with inhomogeneous boundary conditions.
\end{abstract}

Keywords: elliptic operator, non-power degeneration, Gårding inequality, variational Dirichlet problem.

Mathematics Subject Classification: 35J35, 35D05, 35J70, 46E35, 35J40

\section{INTRODUCTION}

It is known in the theory of partial differential equations (see, for insance, [1, 2]) that Gårding inequality 3 plays an important role in studying the solvability of boundary value problems for uniformly elliptic equations by means of the methods of functional analysis. However, the studying of boundary value problems for degenerate elliptic equations by means of the methods of functional analysis was made mostly without using Gårding inequality (see, for instance, [4][10]). In the case of degenerate differential operator, Gårding inequality was proven in works [11, 12. Elliptic operators considered in [11] has a special form, are defined in a bounded domain $\Omega^{+}$located in the half-space $E_{n+1}^{+}=\left\{(x, y)=\left(x_{1}, x_{2}, \ldots, x_{n}, y\right): y>0\right\}$ and adjacent to the hyperplane $y=0$. Instead of usual differentiation operators, their definitions involved the operators of the form

$$
\widetilde{D}^{m+r}=D_{x}^{m} \widetilde{D}_{y}^{r}, \quad D_{x}^{m}=\frac{\partial^{|m|}}{\partial x_{1}^{m_{1}} \cdots \partial x_{n}^{m_{n}}}, \quad \widetilde{D}_{y}^{r}=y^{r} \frac{\partial^{r}}{(y \partial y)^{r}},
$$

and as a result, the degeneration holds only on a part $\Gamma^{0}$ of the boundary of domain $\Omega^{+}$lying in the hyperplane $y=0$. Elliptic operators considered in [12] are defined in arbitrary (bounded or unbounded) domain and have the same degeneration in all independent variables.

In contrast to works [11, 12, here we consider general higher order elliptic operators in an arbitrary (bounded or unbounded) domain with different characters of degeneration in different independent variables.

S.A. Iskhokov, M.G. Gadoev, I.A. Yakushev, GÅrding inequality for higher order elliptic OPERATORS WITH A NON-POWER DEGENERATION AND ITS APPLICATIONS.

(c) Iskhokov S.A., Gadoev M.G., Yakushev I.A. 2016.

Submitted May 12, 2015. 
A part of the results of the present paper in a brief form without proofs was announced in [13.

\section{Functional spaces. Auxiliary integral inequalities}

Let $R_{n}$ be an $n$-dimensional Euclidean space and let $\Pi(0)=\left\{x=\left(x_{1}, \ldots, x_{n}\right) \in R_{n}:\left|x_{i}\right|<\right.$ $1 / 2, i=\overline{1, n}\}$ be the unit cube centered at the origin. For each point $\xi \in R_{n}$ and each vector $\vec{t}=\left(t_{1}, \ldots, t_{n}\right)$ with positive components we define a parallelepiped $\Pi_{\vec{t}}(\xi)$ by the identity

$$
\Pi_{\vec{t}}(\xi)=\left\{x \in R_{n}:\left(\left(x_{1}-\xi_{1}\right) / t_{1}, \ldots,\left(x_{n}-\xi_{n}\right) / t_{n}\right) \in \Pi(0)\right\} .
$$

Let $\Omega$ be an arbitrary open domain in $R_{n}$ and let $g_{i}(x)(i=\overline{1, n})$ be positive functions defined in $\Omega$. We let $\Pi_{\varepsilon, \vec{g}}(\xi)=\Pi_{\varepsilon \cdot \vec{g}(\xi)}(\xi)$, where $\vec{g}(\xi)=\left(g_{1}(\xi), \ldots, g_{n}(\xi)\right)$.

Hereafter we assume that set $\Omega$ and functions $g_{i}(x)(i=\overline{1, n})$ are related by the condition: there exists a number $\varepsilon_{0}>0$ such that for each $\xi \in \Omega$ the parallelepiped $\Pi_{\varepsilon_{0}, \vec{g}}(\xi)$ is contained in $\Omega$. This condition is an analogue of the immersion condition considered in work by P.I. Lizorkin [14]. In [14], there were also considered examples of domains $\Omega$ and positive functions $g_{i}(x)$ $(i=\overline{1, n})$ satisfying the immersion condition.

Let $\sigma(x)$ be a positive function defined in $\Omega$. We assume that for each $\varepsilon \in\left(0, \varepsilon_{0}\right)$ there exist positive numbers $\lambda(\varepsilon), \nu(\varepsilon)$ such that

$$
\lim _{\varepsilon \rightarrow 0+} \lambda(\varepsilon)=\lim _{\varepsilon \rightarrow 0+} \nu(\varepsilon)=1
$$

and

$$
\frac{1}{\nu(\varepsilon)} \leqslant \frac{\sigma(x)}{\sigma(\xi)} \leqslant \nu(\varepsilon), \frac{1}{\lambda(\varepsilon)} \leqslant \frac{g_{i}(x)}{g_{i}(\xi)} \leqslant \lambda(\varepsilon), i=\overline{1, n}
$$

for all $x \in \Pi_{\varepsilon, \vec{g}}(\xi)$ and all $\xi \in \Omega$.

We denote by $\Phi_{\varepsilon, \vec{g}}(\xi)$ the class of positive functions $\sigma(x), x \in \Omega$, satisfying condition (2.1).

Let $1 \leqslant p<+\infty$ and $r$ be a natural number. By the symbol $L_{p, r}^{s}(\Omega ; \sigma, \vec{g})$, where an integer number $s$ is so that $0 \leqslant s \leqslant r$, we denote the class of functions $u(x), x \in \Omega$ having Sobolev generalized derivatives $u^{(k)}(x), k=\left(k_{1}, k_{2} \ldots, k_{n}\right)$ is a multi-index, $|k|=k_{1}+k_{2} \cdots+k_{n} \leqslant r$, with a finite semi-norm

$$
\left\|u ; L_{p, r}^{s}(\Omega ; \sigma, \vec{g})\right\|=\left\{\sum_{|k|=s} \int_{\Omega}\left(\sigma(x) g_{1}^{k_{1}-r}(x) g_{2}^{k_{2}-r}(x) \ldots g_{n}^{k_{n}-r}(x)\left|u^{(k)}(x)\right|\right)^{p} d x\right\}^{1 / p}
$$

while by the symbol $W_{p}^{r}(\Omega ; \sigma, \vec{g})$ we denote the space of functions $u \in L_{p, r}^{r}(\Omega ; \sigma, \vec{g})$ with a finite norm

$$
\left\|u ; W_{p}^{r}(\Omega ; \sigma, \vec{g})\right\|=\left\{\left\|u ; L_{p, r}^{r}(\Omega ; \sigma, \vec{g})\right\|^{p}+\left\|u ; L_{p, r}^{0}(\Omega ; \sigma, \vec{g})\right\|^{p}\right\}^{1 / p} .
$$

Space $W_{p}^{r}(\Omega ; \sigma, \vec{g})$ is a Banach one with norm $(2.2)$ and under the above assumptions for all $p \in[1, \infty)$ and all natural $r$ set $C_{0}^{\infty}(\Omega)$ is dense in this space [10].

By the symbol $L_{p}(\Omega ; \sigma)$ we denote a weighted Lebesgue space with the norm

$$
\left\|u ; L_{p}(\Omega ; \sigma)\right\|=\left\{\int_{\Omega} \sigma^{p}(x)|u(x)|^{p} d x\right\}^{1 / p} .
$$

For each natural number $m$ and each $\varepsilon>0$ we denote by $\Pi_{\varepsilon, \vec{g}}^{(m)}(\xi)$ the parallelepiped $\Pi_{\varepsilon_{m}, \vec{g}}(\xi)$ as $\varepsilon_{m}=m \cdot \varepsilon /(m+1)$. We note that $\Pi_{\varepsilon, \vec{g}}^{(m)}(\xi) \subset \Pi_{\varepsilon_{0}, \vec{g}}(\xi), \varepsilon \in\left(0, \varepsilon_{0}\right)$, for each natural number $m$.

The next lemma (see Lemma 2 of work [10]) is the generalization of the well-known Troizi lemma [15] for the considered case and it is used while obtaining weighted integral inequalities by means of non-weighted ones. 
Lemma 2.1 ([10]). Under the above assumptions for domain $\Omega$ and for positive functions $\sigma(x), g_{i}(x), x \in \Omega, i=\overline{1, n}$, the equivalence relation

$$
\int_{\Omega}\left(\sigma(\xi)\left\|u ; L_{p}\left(\Pi_{\varepsilon, \vec{g}}(\xi)\right)\right\|\right)^{p} d \xi \asymp \int_{\Omega}\left(\sigma(x)\left(g_{1}(x) g_{2}(x) \ldots g_{n}(x)\right)^{1 / p}|u(x)|\right)^{p} d x
$$

holds true, where the symbol $\asymp$ stands for a two-sided estimate with some positive constant.

Lemma 2.2. Let $\chi_{\varepsilon, \vec{g}}^{(m)}(x ; \xi)$ be a characteristic function of parallelepiped $\Pi_{\varepsilon, \vec{g}}^{(m)}(\xi)$. Then for each natural number $m$ and sufficiently small $\varepsilon>0$ the inequality

$$
\left(\frac{\varepsilon}{2 \lambda(\varepsilon)}\right)^{n} \leqslant\left(1+\frac{1}{m}\right)^{n} g_{1}^{-1}(x) g_{2}^{-1}(x) \ldots g_{n}^{-1}(x) \int \chi_{\varepsilon, \vec{g}}^{(m)}(x ; \xi) d \xi \leqslant\left(\frac{\lambda(\varepsilon) \varepsilon}{2}\right)^{n}
$$

holds true, where $\lambda(\varepsilon)$ is the same as in condition (2.1).

Proof. For an arbitrary fixed point $x \in \Omega$ we introduce the following notations:

$$
\begin{aligned}
& T_{\varepsilon, \vec{g}}(x)=\left\{\xi \in R_{n}:\left|\xi_{i}-x_{i}\right|<\frac{\varepsilon}{2} g_{i}(\xi), i=\overline{1, n}\right\}, \\
& D_{\varepsilon, \vec{g}}(x)=\left\{\xi \in R_{n}:\left|\xi_{i}-x_{i}\right|<\frac{\varepsilon}{2} g_{i}(x), i=\overline{1, n}\right\} .
\end{aligned}
$$

Let $\xi \in D_{\varepsilon \lambda(\varepsilon)^{-1, \vec{g}}}(x)$. Then it follows from condition $(2.1)$ that $\left|\xi_{i}-x_{i}\right|<\varepsilon \cdot g_{i}(x) / 2 \lambda(\varepsilon)<$ $<\varepsilon g_{i}(\xi) / 2, i=\overline{1, n}$. Therefore, $\xi \in T_{\varepsilon, \vec{g}}(x)$, and this is why $D_{\varepsilon \lambda(\varepsilon)^{-1}, \vec{g}}(x) \subset T_{\varepsilon, \vec{g}}(x)$ for all $x \in \Omega$. In the same way by condition $(2.1)$ we prove that $T_{\varepsilon, \vec{g}}(x) \subset D_{\varepsilon \lambda(\varepsilon), \vec{g}}(x)$ for each $x \in \Omega$.

Let $\chi_{\varepsilon, \vec{g}}(x, \xi)$ be a characteristic function of parallelepiped $\Pi_{\varepsilon, \vec{g}}(\xi)$. Since $\int \chi_{\varepsilon, \vec{g}}(x, \xi) d \xi=$ $\left|T_{\varepsilon, \vec{g}}(x)\right|$, where the right hand side denotes the volume of parallelepiped $T_{\varepsilon, \vec{g}}(x)$, the above inclusions imply that

$$
\left|D_{\varepsilon \lambda(\varepsilon)^{-1}, \vec{g}}(x)\right| \leqslant \int \chi_{\varepsilon, \vec{g}}(x, \xi) d \xi \leqslant\left|D_{\varepsilon \lambda(\varepsilon), \vec{g}}(x)\right|
$$

Hence,

$$
\left(\frac{\varepsilon}{2 \lambda(\varepsilon)}\right)^{n} \leqslant g_{1}^{-1}(x) \cdot g_{2}^{-1}(x) \ldots g_{n}^{-1}(x) \int \chi_{\varepsilon, \vec{g}}(x, \xi) d \xi \leqslant\left(\frac{\lambda(\varepsilon) \varepsilon}{2}\right)^{n} .
$$

Replacing $\varepsilon$ by $\varepsilon_{m}=\varepsilon m /(m+1)$ in this inequality, we obtain (2.3).

Lemma 2.3. Suppose that an integer number s satisfies $0 \leqslant s<r$. Let $p \geqslant 1,1 \leqslant q_{1} \leqslant q_{0}$ satisfy the conditions

$$
\frac{1}{p}-\frac{r-s}{n}<\frac{1}{q_{0}} \quad \text { as } \quad n-(r-s) p>0
$$

$q_{0}$ is an arbitrary finite number as $n-(r-s) p \leqslant 0$.

Then for each $\tau>0$ and all $v \in W_{p}^{r}(\Omega ; \sigma, \vec{g})$ the inequality

$$
\begin{aligned}
\left\|v ; L_{q_{0}, r}^{s}\left(\Omega ; \sigma\left(g_{1} g_{2} \ldots g_{n}\right)^{\frac{1}{p}-\frac{1}{q_{0}}}, \vec{g}\right)\right\| \leqslant & \tau\left\|v ; W_{p}^{r}(\Omega ; \sigma, \vec{g})\right\| \\
& +c_{1} \tau^{-\mu}\left\|v ; L_{q_{1}}\left(\Omega ; \sigma\left(g_{1} g_{2} \ldots g_{n}\right)^{-r+\frac{1}{p}-\frac{1}{q_{1}}}, \vec{g}\right)\right\|,
\end{aligned}
$$

holds true, where

$$
\mu=\frac{q_{1}^{-1}-q_{0}^{-1}+s n^{-1}}{q_{0}^{-1}-p^{-1}+(r-s) n^{-1}} .
$$

Proof. Under the assumptions of the lemma, it follows from the interpolation inequalities for classical Sobolev space (see, for instance, [16, Sect. 4.7]) that

$$
\left\|u^{(k)} ; L_{q_{0}}(\Pi(0))\right\| \leqslant \tau \sum_{|l|=r}\left\|u^{(l)} ; L_{p}(\Pi(0))\right\|+\tau\left\|u ; L_{p}(\Pi(0))\right\|+c_{1} \tau^{-\mu}\left\|u ; L_{q_{1}}(\Pi(0))\right\|,
$$


where $k$ is an arbitrary multi-index of length $s$ and number $\mu$ is determined by identity (2.5).

Inequality (2.4) can be obtained from (2.6) by applying Lemma 2.1 and employing the technique used in the proof of Lemma 2.2 in work [12].

Similarly to Lemma 2.3 , one can prove that if number $s$ is so that $0 \leqslant s \leqslant r$ and the conditions

$$
1 \leqslant p \leqslant q_{0}<+\infty, r-s-\frac{n}{p}+\frac{n}{q_{0}}>0
$$

hold true, then for each $v \in W_{p}^{r}(\Omega ; \sigma, \vec{g})$ the inequality

$$
\left\|v ; L_{q_{0}, r}^{s}\left(\Omega ; \sigma\left(g_{1} g_{2} \ldots g_{n}\right)^{\frac{1}{p}-\frac{1}{q_{0}}}, \vec{g}\right)\right\| \leqslant M\left\|v ; W_{p}^{r}(\Omega ; \sigma, \vec{g})\right\|
$$

holds true, where number $M>0$ is independent of function $v(x)$.

Lemma 2.4. Assume that positive functions $\alpha(x), \beta(x)$ belong to the class $\Phi_{\varepsilon, \vec{g}}(\Omega), p \geqslant 1$, $q \geqslant 1$ and $r, t$ are natural numbers. For multi-indices $k, l$ such that $|k|<r,|l| \leqslant t$, we define numbers $q_{l}, \lambda_{k l}, s_{k}$ by the following relations:

$$
\begin{aligned}
& \frac{1}{q_{l}}= \begin{cases}\frac{1}{q}-\frac{t-|l|}{n}, & n>q(t-|l|), \\
\varepsilon_{1}, 0<\varepsilon_{1} \leqslant 1 / q, & n \leqslant q(t-|l|),\end{cases} \\
& \frac{1}{\lambda_{k l}}>\frac{1}{q_{l}}+\frac{1}{p}-\frac{r-|k|}{n}, \quad \text { as } n-p(r-|k|)>0, \\
& \frac{1}{\lambda_{k l}}=\frac{1}{q_{l}}+\varepsilon_{2}, \quad \text { where } \quad 0<\varepsilon_{2}<1 / p \text { as } n-p(r-|k|) \leqslant 0, \\
& \frac{1}{s_{k}} \geqslant \frac{1}{\lambda_{k l}}-\frac{1}{q_{l}} .
\end{aligned}
$$

Suppose that a positive function $\sigma_{k l}(x)$ belongs to the class $\Phi_{\varepsilon, \vec{g}}(\Omega)$ and satisfies the inequality

$$
\begin{aligned}
\sigma_{k l}(x) \alpha^{-1}(x) \beta^{-1}(x) \leqslant & c g_{1}^{k_{1}+l_{1}}(x) g_{2}^{k_{2}+l_{2}}(x) \ldots g_{n}^{k_{n}+l_{n}}(x) \\
& \cdot\left(g_{1}(x) g_{2}(x) \ldots g_{n}(x)\right)^{-t+\frac{1}{q}-r+\frac{1}{p}-\frac{1}{\lambda_{k l}}}
\end{aligned}
$$

for each $x \in \Omega$; a positive number $c$ is independent of $x$.

Then for each $\tau>0$ the inequality

$$
\begin{aligned}
\left\|u^{(k)} v^{(l)} ; L_{\lambda_{k l}}\left(\Omega ; \sigma_{k l}\right)\right\| \leqslant & \left\|v ; W_{q}^{t}(\Omega ; \beta, \vec{g})\right\| \cdot\left\{\left\|u ; W_{p}^{r}(\Omega ; \alpha, \vec{g})\right\|\right. \\
& \left.+c_{0} \tau^{-\mu_{k}}\left\|u ; L_{s_{k}}\left(\Omega ; \alpha,\left(g_{1} g_{2} \ldots g_{n}\right)^{-r+\frac{1}{p}-\frac{1}{s_{k}}}\right)\right\|\right\}
\end{aligned}
$$

holds true, where

$$
\mu_{k}=\frac{s_{k}^{-1}-\lambda_{k l}^{-1}+q_{l}^{-1}+|k| n^{-1}}{\lambda_{k l}^{-1}-q_{l}^{-1}-p^{-1}+(r-|k|) n^{-1}}
$$

and a positive constant $c_{0}$ depends only on $n, p, r,|k|$.

Proof. Let $|k|<r,|l| \leqslant t$. Since number $q_{l}$ defined by identity (2.8) satisfies the conditions

$$
1 \leqslant q \leqslant q_{l}<\infty, t-|l|-\frac{n}{q}+\frac{n}{q_{l}}>0,
$$

by applying inequality (2.7) we have

$$
\begin{aligned}
\| v^{(l)} ; L_{q_{l}}\left(\Omega ; \beta(x) g_{1}^{l_{1}}(x) g_{2}^{l_{2}}(x) \ldots g_{n}^{l_{n}}(x)\left(g_{1}(x) g_{2}(x)\right.\right. & \left.\left.\ldots g_{n}(x)\right)^{-t+\frac{1}{q}-\frac{1}{q_{l}}}\right) \| \\
& \leqslant M\left\|v ; W_{q}^{t}(\Omega ; \beta, \vec{g})\right\|,
\end{aligned}
$$

where $M$ is a positive constant independent of $v(x)$. 
We observe that as $q_{0}=p_{k}, s=|k|, q_{1}=s_{k}$, where $p_{k}=\left(\lambda_{k l}^{-1}-q_{l}^{-1}\right)^{-1}$, and numbers $q_{l}, \lambda_{k l}$, $s_{k}$ are defined by relations (2.8)-(2.11) and the assumptions of Lemma 2.3. Hence, applying Lemma 2.3, in this case we obtain

$$
\begin{aligned}
\left\|u^{(k)} ; L_{p_{k}}\left(\Omega ; \alpha g_{1}^{k_{1}} g_{2}^{k_{2}} \ldots g_{n}^{k_{n}}\left(g_{1} g_{2} \ldots g_{n}\right)^{-r+\frac{1}{p}-\frac{1}{p_{k}}}\right)\right\| \leqslant \tau\left\|u ; W_{p}^{r}(\Omega ; \alpha, \vec{g})\right\| \\
\quad+c_{0} \tau^{-\mu_{k}}\left\|u ; L_{s_{k}}\left(\Omega ; \alpha\left(g_{1} g_{2} \ldots g_{n}\right)^{-r+\frac{1}{p}-\frac{1}{s_{k}}}\right)\right\|
\end{aligned}
$$

where

$$
\mu_{k}=\frac{s_{k}-p_{k}^{-1}+|k| n^{-1}}{p_{k}^{-1}-p^{-1}+(r-|k|) n^{-1}} .
$$

By the identity

$$
\frac{1}{\lambda_{k l}}=\frac{1}{p_{k}}+\frac{1}{q_{l}}
$$

condition 2.12 and Hölder inequality we prove that

$$
\begin{aligned}
\left\|u^{(k)} v^{(l)} ; L_{\lambda_{k l}}\left(\Omega ; \sigma_{k l}\right)\right\| \leqslant & \left\|v^{(l)} ; L_{q_{l}}\left(\Omega ; \beta g_{1}^{l_{1}} g_{2}^{l_{2}} \ldots g_{n}^{l_{n}}\left(g_{1} g_{2} \ldots g_{n}\right)^{-t+\frac{1}{q}-\frac{1}{q_{l}}}\right)\right\| \\
& \cdot\left\|u^{(k)} ; L_{q_{k}}\left(\Omega ; \alpha g_{1}^{k_{1}} g_{2}^{k_{2}} \ldots g_{n}^{k_{n}}\left(g_{1} g_{2} \ldots g_{n}\right)^{-r+\frac{1}{p}-\frac{1}{p_{k}}}\right)\right\| .
\end{aligned}
$$

Now it is easy to see that $(2.15),(2.16),(2.19)$ imply inequality $(2.13)$. Identity $(2.14)$ is implied by 2.17$)$ and identity $(2.18)$.

\section{GÅRING INEQUALITY FOR DEGENERATE ELLIPTIC OPERATORS}

We consider a differential operator

$$
(A u)(x)=\sum_{|k|,|l| \leqslant r}(-1)^{|l|}\left(p_{k}(x) p_{l}(x) a_{k l}(x) u^{(k)}(x)\right)^{(l)}, \quad x \in \Omega,
$$

where

$$
p_{k}(x)=\sigma(x) g_{1}^{-r+k_{1}}(x) g_{2}^{-r+k_{2}}(x) \ldots g_{n}^{-r+k_{n}}(x)
$$

and $a_{k l}(x)$ are complex-valued functions.

The studying of boundary value problems for the differential equations with differential operator (3.1) by the methods of functional analysis is related with the sesquilinear form generated by this operator

$$
B[u, v]=\sum_{|k|,|l| \leqslant r} \int_{\Omega} p_{k}(x) p_{l}(x) a_{k l}(x) u^{(k)}(x) \overline{v^{(l)}(x)} d x .
$$

The Dirichlet variational problem related with form (3.3) was studied earlier in work by S.A. Iskhokov [10] under the assumption that coefficients satisfy the following ellipticity condition

$$
\operatorname{Re} \sum_{|k|,|l| \leqslant r} a_{k l}(x) \zeta_{k} \overline{\zeta_{l}} \geqslant c \sum_{|k|=r}\left|\zeta_{k}\right|^{2}
$$

for each $x \in \Omega$ and each set of complex numbers $\zeta=\left\{\zeta_{k}\right\}_{|k| \leqslant r}$. Number $c>0$ is independent of $x, \zeta$. In this section instead of condition (3.4) we assume a weaker condition

$$
\operatorname{Re} \sum_{|k|,|l|=r} a_{k l}(x) \xi^{k} \xi^{l} \geqslant c|\xi|^{2 r}
$$

for each $x \in \Omega, \xi \in R_{n} ; \xi^{k}=\xi_{1}^{k_{1}} \xi_{2}^{k_{2}} \cdots \xi_{n}^{k_{n}}, c$ is a positive constant independent of $x, \xi$. 
Theorem 3.1. Suppose the coefficients $a_{k l}(x)$ are bounded as $|k|=|l|=r$, satisfy ellipticity condition (3.5) and for each sufficiently small number $\nu>0$ there exists a number $\varepsilon>0$ such that

$$
\left|a_{k l}(y)-a_{k l}(z)\right|<\nu
$$

for each $y \in \Omega$ and each

$$
z \in \Pi_{\varepsilon, \vec{g}}(y)=\left\{z \in R_{n}:\left|z_{i}-y_{i}\right|<\frac{1}{2} \varepsilon g_{i}(y), i=\overline{1, n}\right\}
$$

Assume also that coefficients $a_{k l}(x)$ belong to the space $L_{p_{k l}}\left(\Omega ;\left(g_{1} \cdot g_{2} \ldots g_{n}\right)^{-1 / p_{k l}}\right)$ as $|k|,|l| \leqslant r$ $|k|+|l| \leqslant 2 r-1$, where

$$
p_{k l}= \begin{cases}q_{k l} & \text { as }|k| \leqslant r-1,|l| \leqslant r \\ q_{l k} & \text { as }|k|=r,|l| \leqslant r-1\end{cases}
$$

while numbers $q_{k l}$ are determined by the relations:

$$
\begin{aligned}
& \frac{n}{2 r-|k|-|l|}<q_{k l} \leqslant \frac{n}{r-|l|}, \quad \text { if } n>2(r-|k|), n>2(r-|l|) ; \\
& \frac{n}{r-|k|-\varepsilon_{1} n}<q_{k l}, 0<\varepsilon_{1}<\frac{1}{2}, \quad \text { if } \quad n>2(r-|k|), n \leqslant 2(r-|l|) \text {; } \\
& q_{k l}= \begin{cases}\frac{n}{r-|l|+\varepsilon_{2} n}, 0<\varepsilon_{2}<\frac{1}{2}, & \text { if } n \leqslant 2(r-|k|), n>2(r-|l|), \\
\text { arbitrary number }>1 & \text { if } n \leqslant 2(r-|k|), \quad n \leqslant 2(r-|l|) .\end{cases}
\end{aligned}
$$

Then there exist constant $c_{1}>0$ and $c_{2} \geqslant 0$ such that

$$
\operatorname{Re} B[u, u] \geqslant c_{1}\left\|u ; W_{2}^{r}(\Omega ; \sigma, \vec{g})\right\|^{2}-c_{2}\left\|u ; L_{2, r}^{0}(\Omega ; \sigma, \vec{g})\right\|^{2}
$$

for each $u \in W_{2}^{r}(\Omega ; \sigma, \vec{g})$.

Proof. First we consider the case when sesquilinear form 3.3 contains no lower order terms, i.e., as $a_{k l}(x) \equiv 0(x \in \Omega)$ for all multi-indices $k, l$ such that $|k|,|l| \leqslant r$ and $|k|+|l| \leqslant 2 r-1$.

Fixing arbitrary point $y \in \Omega$, we consider the sesquilinear form

$$
B_{y}[u, v]=\sum_{|k|=|l|=r} \int_{R_{n}} a_{k l}(y) u^{(k)}(x) \overline{v^{(l)}(x)} d x, u, v \in C_{0}^{\infty}\left(R_{n}\right) .
$$

Applying Gårding inequality for strongly elliptic operators with constant coefficients, we have

$$
\sum_{|k|=r} \int_{R_{n}}\left|u^{(k)}(x)\right|^{2} d x \leqslant M\left\{\operatorname{Re} \sum_{|k|=|l|=r} \int_{R_{n}} a_{k l}(y) u^{(k)}(x) \overline{u^{(l)}(x)} d x+\int_{R_{n}}|u(x)|^{2} d x\right\}
$$

for each $u \in C_{0}^{\infty}\left(R_{n}\right)$.

We introduce the notation

$$
\Pi_{m}(0)=\left\{x=\left(x_{1}, x_{2}, \ldots, x_{n}\right) \in R_{n}:\left|x_{i}\right|<\frac{m}{2(m+1)}, i=\overline{1, n}\right\},
$$

where $m$ is a natural number.

We take a function $\varphi_{m}(x) \in C_{0}^{\infty}\left(\Pi_{2 m}(0)\right)$ with the following properties:

1) $0 \leqslant \varphi_{m}(x) \leqslant 1$ for each $x \in \Pi(0)$;

2) $\varphi_{m}(x)=1$ for each $x \in \Pi_{m}(0)$;

3) there exists a number $c>0$ such that $\left|\varphi_{m}^{(k)}(x)\right| \leqslant c$ for each $x \in \Pi(0)$ and each multi-index $k:|k| \leqslant r$. 
Let $u(x)$ be an arbitrary function in the class $C^{\infty}(\Pi(0))$. Continuing function $v_{m}(x)=$ $u(x) \varphi_{m}(x)$ by zero outside set $\Pi(0)$, we obtain function $v_{m} \in C_{0}^{\infty}\left(R_{n}\right)$. Since $v_{m}(x)=u(x)$ for all $x \in \Pi_{m}(0)$, inequality 3.8 for function $v_{m}(x)$ implies that

$$
\sum_{|k|=r} \int_{\Pi_{m}(0)}\left|u^{(k)}(x)\right|^{2} d x \leqslant M_{0}\left\{\operatorname{Re} B_{y}\left[v_{m}, v_{m}\right]+\int_{\Pi(0)}|u(x)|^{2} d x\right\} .
$$

We represent form $B_{y}\left[v_{m}, v_{m}\right]$ as

$$
B_{y}\left[v_{m}, v_{m}\right]=B_{y}^{(1)}\left[v_{m}, v_{m}\right]+B_{y}^{(2)}\left[v_{m}, v_{m}\right],
$$

where

$$
\begin{aligned}
& B_{y}^{(1)}\left[v_{m}, v_{m}\right]=\sum_{|k|=|l|=r} \int_{\Pi(0)} a_{k l}(y) \varphi_{m}^{2}(x) u^{(k)}(x) \overline{u^{(l)}(x)} d x, \\
& B_{y}^{(2)}\left[v_{m}, v_{m}\right]=B_{y}\left[v_{m}, v_{m}\right]-B_{y}^{(1)}\left[v_{m}, v_{m}\right] .
\end{aligned}
$$

Since in all the integrals involved in sesquilinear form $B_{y}^{(2)}\left[v_{m}, v_{m}\right]$ the order of at least one of the derivatives $u^{(k)}(x), u^{(l)}(x)$ does not exceed $r-1$, applying appropriate embedding theorems for Sobolev spaces without weight as well as Young inequality with a small parameter, we obtain that for each sufficiently small $\tau>0$ there exists a finite number $M(\tau)>0$ such that

$$
\left|B_{y}^{(2)}\left[v_{m}, v_{m}\right]\right| \leqslant \tau \sum_{|k|=r} \int_{\Pi(0)}\left|u^{(k)}(x)\right|^{2} d x+M(\tau) \int_{\Pi(0)}|u(x)|^{2} d x .
$$

Indeed, the integrals involved in form $B_{y}^{(2)}\left[v_{m}, v_{m}\right]$ have the following general form

$$
I_{y, k, l, \mu, \nu}^{(m)}(u)=\int_{\Pi(0)} C_{k, \mu} C_{l, \nu} a_{k l}(y) \varphi_{m}^{(\mu)}(x) u^{(k-\mu)}(x) \varphi_{m}^{(\nu)}(x) \overline{u^{(l-\nu)}(x)} d x
$$

where $|\mu|+|\nu| \neq 0$, and by property 3) of functions $\varphi_{m}$ and the boundedness of coefficients $a_{k l}$, $|k|=|l|=r$, they satisfy the inequality

$\left|I_{y, k, l, \mu, \nu}^{(m)}(u)\right| \leqslant M_{0} \int_{\Pi(0)}\left|u^{(k-\mu)}(x)\right| \cdot\left|u^{(l-\nu)}(x)\right| d x \leqslant M_{0}\left\|u^{(k-\mu)} ; L_{2}(\Pi(0))\right\| \cdot\left\|u^{(l-\nu)} ; L_{2}(\Pi(0))\right\|$.

Applying Young inequality

$$
|a b| \leqslant \delta|a|^{2}+\frac{1}{4 \delta}|b|^{2}
$$

where $\delta$ is a sufficiently small positive number, and interpolation inequality (2.6), one can show that $\left|I_{y, k, l, \mu, \nu}^{(m)}(u)\right|$ does not exceed the right hand side of inequality 3.11 .

In view of property 2 ) of functions $\varphi_{m}(x)$, we represent form $B_{y}^{(1)}\left[v_{m}, v_{m}\right]$ as

$$
B_{y}^{(1)}\left[v_{m}, v_{m}\right]=B_{y, m}^{(11)}[u, u]+B_{y, m}^{(12)}[u, u],
$$

where

$$
\begin{aligned}
& B_{y, m}^{(11)}[u, u]=\sum_{|k|=|l|=r_{\Pi_{m}(0)}} \int_{k l}(y) u^{(k)}(x) \overline{u^{(l)}(x)} d x, \\
& B_{y, m}^{(12)}[u, u]=\sum_{|k|=|l|=\Pi_{\Pi^{(m)}(0)}} \int_{k l} a_{k l}(y) \varphi_{m}^{2}(x) u^{(k)}(x) \overline{u^{(l)}(x)} d x, \\
& \Pi^{(m)}(0)=\Pi(0) \backslash \Pi_{m}(0)=\left\{x \in R_{n}: \frac{m}{2(m+1)}<\left|x_{i}\right|<\frac{1}{2}, i=\overline{1, n}\right\} .
\end{aligned}
$$


Since coefficients $a_{k l}(|k|=|l|=r)$ are bounded, applying Cauchy-Schwarz inequality and taking into consideration that $\left|\Pi^{(m)}(0)\right| \rightarrow 0$ as $m \rightarrow \infty$, we obtain

$$
\left|B_{y, m}^{(12)}[u, u]\right| \leqslant \mu_{m}\left\|u ; L_{2}^{r}(\Pi(0))\right\|^{2}
$$

where

$$
\left\|u ; L_{2}^{r}(\Pi(0))\right\|=\left\{\sum_{|k|=r} \int_{\Pi(0)}\left|u^{(k)}(x)\right|^{2} d x\right\}^{1 / 2}
$$

and positive numbers $\mu_{m}$ tend to zero as $m \rightarrow \infty$.

By inequality (3.9) it follows from representations (3.10), (3.12) that

$$
\left\|u ; L_{2}^{r}\left(\Pi_{m}(0)\right)\right\|^{2}-M_{0}\left|B_{y}^{(2)}\left[v_{m}, v_{m}\right]\right|-M_{0}\left|B_{y, m}^{(12)}[u, u]\right| \leqslant M_{0} \operatorname{Re} B_{y, m}^{(11)}[u, u] .
$$

Choosing natural number $m$ large enough and applying inequality 3.11 as $\tau=\frac{1}{m}$ as well as inequality (3.13), we arrive at the inequality

$$
\left\|u ; L_{2}^{r}\left(\Pi_{m}(0)\right)\right\|^{2}-c_{m}\left\|u ; L_{2}^{r}(\Pi(0))\right\|^{2}-\mathbb{C}_{m}\left\|u ; L_{2}(\Pi(0))\right\|^{2} \leqslant M_{0} \operatorname{Re} B_{y, m}^{(11)}[u, u]
$$

for all $u \in C^{\infty}(\Pi(0))$, where $c_{m}, \mathbb{C}_{m}$ are positive numbers independent of $u(x)$ and $c_{m} \rightarrow 0$ as $m \rightarrow \infty$.

Let $v$ be an arbitrary function in class $C_{0}^{\infty}(\Omega)$ and $y$ be an arbitrary fixed point in domain $\Omega$. Mapping $z \rightarrow x$ defined by the identities $x_{i}=\left(z_{i}-y_{i}\right) /\left(\varepsilon g_{i}(y)\right), i=\overline{1, n}$, maps the parallelepiped $\Pi_{\varepsilon, \vec{g}}(y)=\left\{z \in R_{n}:\left|z_{i}-y_{i}\right|<\varepsilon g_{i}(y) / 2\right\}$ into the unit cube $\Pi(0)$, while parallelepiped $\Pi_{\varepsilon, \vec{g}}^{(m)}(y)$ is mapped into $\Pi_{m}(0)$. For sufficiently small $\varepsilon>0$ the parallelepiped $\Pi_{\varepsilon, \vec{g}}(y)$ is contained in domain $\Omega$ and this is why function $\hat{v}_{y}(x)=v\left(x_{i} \varepsilon g_{i}(y)+y_{i}\right)$ is well-defined for all $x \in \Pi(0)$ and belongs to class $C^{\infty}(\Pi(0))$.

Inequality (3.14) for the function $u(x)=\hat{v}_{y}(x)$ becomes

$$
\begin{gathered}
\sum_{|k|=r}\left\{\int_{\Pi_{m}(0)}\left|\hat{v}_{y}^{(k)}(x)\right|^{2} d x-c_{m} \int_{\Pi(0)}\left|\hat{v}_{y}^{(k)}(x)\right|^{2} d x\right\} \varepsilon^{2 r} g_{1}^{2 k_{1}}(y) g_{2}^{2 k_{2}}(y) \ldots g_{n}^{2 k_{n}}(y)-\mathbb{C}_{m} \int_{\Pi(0)}\left|\hat{v}_{y}(x)\right|^{2} d x \\
\leqslant M_{0} \operatorname{Re} \sum_{|k|=|l|=r} \varepsilon^{2 r} g_{1}^{k_{1}+l_{1}}(y) g_{2}^{k_{2}+l_{2}}(y) \ldots g_{n}^{k_{n}+l_{n}}(y) \int_{\Pi_{m}(0)} a_{k l}(y) \hat{v}_{y}^{(k)}(x) \overline{v_{y}^{(l)}(x)} d x
\end{gathered}
$$

We pass to new integration variables $z_{i}=x_{i} \varepsilon g_{i}(y)+y_{i}$ in the above integrals to obtain

$$
\begin{aligned}
& \sum_{|k|=r}\left\{\int_{\Pi_{\varepsilon, \vec{g}}^{(m)}(y)}\left|v^{(k)}(z)\right|^{2} d z-c_{m} \int_{\Pi_{\varepsilon, \vec{g}}(y)}\left|v^{(k)}(z)\right|^{2} d z\right\} \varepsilon^{2 r-n} g_{1}^{2 k_{1}-1}(y) g_{2}^{2 k_{2}-1}(y) \ldots g_{n}^{2 k_{n}-1}(y) \\
& -\mathbb{C}_{m} \int_{\Pi_{\varepsilon, \vec{g}}(y)}|v(z)|^{2} d z \varepsilon^{-n} g_{1}^{-1}(y) g_{2}^{-1}(y) \ldots g_{n}^{-1}(y) d z \\
& \leqslant M_{0} \operatorname{Re}\left\{\varepsilon^{2 r-n} \sum_{|k|=|l|=r} g_{1}^{k_{1}+l_{1}-1}(y) g_{2}^{k_{2}+l_{2}-1}(y) \ldots g_{n}^{k_{n}+l_{n}-1}(y) \int_{\Pi_{\varepsilon, \vec{g}}^{(m)}(y)} a_{k l}(y) v^{(k)}(z) \overline{v^{(l)}(z) d z}\right\} .
\end{aligned}
$$


We multiply this inequality by $\sigma^{2}(y)\left(g_{1}(y) g_{2}(y) \ldots g_{n}(y)\right)^{-2 r}$ and we integrate the result in $y \in \Omega$. As a result we have

$$
\begin{aligned}
\sum_{|k|=r} \int_{\Omega} \sigma^{2}(y)\left(g_{1}(y) g_{2}(y) \ldots g_{n}(y)\right)^{-2 r} \varepsilon^{2 r-n} g_{1}^{2 k_{1}-1}(y) g_{2}^{2 k_{2}-1}(y) \ldots g_{n}^{2 k_{n}-1}(y) \\
\cdot\left(\int_{\Pi_{\varepsilon, \vec{g}}^{(m)}(y)}\left|v^{(k)}(z)\right|^{2} d z\right) d y \\
-c_{m} \sum_{|k|=r} \int_{\Omega} \sigma^{2}(y)\left(g_{1}(y) g_{2}(y) \ldots g_{n}(y)\right)^{-2 r} \varepsilon^{2 r-n} g_{1}^{2 k_{1}-1}(y) g_{2}^{2 k_{2}-1}(y) \ldots g_{n}^{2 k_{n}-1}(y) \\
\cdot\left(\int_{\Pi_{\varepsilon, \vec{g}}(y)}\left|v^{(k)}(z)\right|^{2} d z\right) d y \\
\quad-\mathbb{C}_{m} \int_{\Omega} \sigma^{2}(y)\left(g_{1}(y) g_{2}(y) \ldots g_{n}(y)\right)^{-2 r-1} \varepsilon^{-n}\left(\int_{\Pi_{\varepsilon, \vec{g}}(y)}\left|v^{(k)}(z)\right|^{2} d z\right) d y \\
\leqslant M_{0} \varepsilon^{2 r-n} \operatorname{Re} B_{\varepsilon, m}[v, v],
\end{aligned}
$$

where

$$
\begin{gathered}
B_{\varepsilon, m}[v, v]=\sum_{|k|=|l|=r} \int_{\Omega} \sigma^{2}(y)\left(g_{1}(y) g_{2}(y) \ldots g_{n}(y)\right)^{-2 r-1} g_{1}^{k_{1}+l_{1}}(y) g_{2}^{k_{2}+l_{2}}(y) \ldots g_{n}^{k_{n}+l_{n}}(y) \\
\cdot\left(\int_{\Pi_{\varepsilon, \vec{g}}^{(m)}(y)} a_{k l}(y) v^{(k)}(z) \overline{v^{(l)}(z)} d z\right) d y .
\end{gathered}
$$

Applying Lemma 2.1 and inequalities (2.1), let us estimate the integrals in the left hand side of inequality (3.15). By means of inequalities (2.1) for the first integral we have

$$
\begin{aligned}
& \varepsilon^{2 r-n} \sum_{|k|=r} \int_{\Omega} \sigma^{2}(y)\left(g_{1}(y) g_{2}(y) \ldots g_{n}(y)\right)^{-2 r-1} g_{1}^{2 k_{1}}(y) g_{2}^{2 k_{2}}(y) \ldots g_{n}^{2 k_{n}}(y) \\
& \cdot\left(\int_{\Pi_{\varepsilon, \vec{g}}^{(m)}(y)}\left|v^{(k)}(z)\right|^{2} d z\right) d y \\
& \geqslant \varepsilon^{2 r-n} \nu^{-2}\left(\varepsilon_{m}\right) \lambda^{-n}\left(\varepsilon_{m}\right) \sum_{|k|=r} \int_{\Omega} \sigma^{2}(z)\left(g_{1}(z) g_{2}(z) \ldots g_{n}(z)\right)^{-2 r-1} g_{1}^{2 k_{1}}(z) g_{2}^{2 k_{2}}(z) \ldots g_{n}^{2 k_{n}}(z) \\
& \cdot\left(\int_{\Omega} \chi_{\varepsilon, \vec{g}}^{(m)}(z ; y) d y\right)\left|v^{(k)}(z)\right|^{2} d z
\end{aligned}
$$

where $\varepsilon_{m}=m \varepsilon /(m+1)$.

Applying Lemma 2.1, we obtain

$$
\begin{aligned}
& \varepsilon^{2 r-n} \sum_{|k|=r} \int_{\Omega} \sigma^{2}(y)\left(g_{1}(y) g_{2}(y) \ldots g_{n}(y)\right)^{-2 r-1} g_{1}^{2 k_{1}}(y) g_{2}^{2 k_{2}}(y) \ldots g_{n}^{2 k_{n}}(y) \\
& \cdot\left(\int_{\Pi_{\varepsilon, \vec{g}}^{(m)}(y)}\left|v^{(k)}(z)\right|^{2} d z\right) d y \geqslant \varepsilon^{2 r} \nu^{-2}\left(\varepsilon_{m}\right) \lambda^{-2 n}\left(\varepsilon_{m}\right) 2^{-n}\left(1+\frac{1}{m}\right)^{-n}\left\|v ; L_{2, r}^{r}(\Omega ; \sigma, \vec{g})\right\|^{2} .
\end{aligned}
$$


Let us estimate the second integral in the left hand side of inequality (3.15). Applying inequalities (2.1), we have

$$
\begin{gathered}
\sum_{|k|=r} \int_{\Omega} \sigma^{2}(y)\left(g_{1}(y) g_{2}(y) \ldots g_{n}(y)\right)^{-2 r} \varepsilon^{2 r-n} g_{1}^{2 k_{1}-1}(y) g_{2}^{2 k_{2}-1}(y) \ldots g_{n}^{2 k_{n}-1}(y) \\
\cdot\left(\int_{\Pi_{\varepsilon, \vec{g}}(y)}\left|v^{(k)}(z)\right|^{2} d z\right) d y \\
\leqslant \varepsilon^{2 r-n} \nu^{2}(\varepsilon) \lambda^{-n}(\varepsilon) \sum_{|k|=r} \int_{\Omega} \sigma^{2}(z) g_{1}^{2 k_{1}-2 r-1}(z) g_{2}^{2 k_{2}-2 r-1}(z) \ldots g_{n}^{2 k_{n}-2 r-1}(z) \\
\cdot\left|v^{(k)}(z)\right|^{2}\left(\int_{\Omega} \chi_{\varepsilon, \vec{g}}(z ; y) d y\right) d z
\end{gathered}
$$

Employing then Lemma 2.1, we obtain the following final estimate

$$
\begin{gathered}
\sum_{|k|=r} \int_{\Omega} \sigma^{2}(y)\left(g_{1}(y) g_{2}(y) \ldots g_{n}(y)\right)^{-2 r} \varepsilon^{2 r-n} g_{1}^{2 k_{1}-1}(y) g_{2}^{2 k_{2}-1}(y) \ldots g_{n}^{2 k_{n}-1}(y) \\
\left(\int_{\Pi_{\varepsilon, \vec{g}}(y)}\left|v^{(k)}(z)\right|^{2} d z\right) d y \leqslant \varepsilon^{2 r} \nu^{2}(\varepsilon) 2^{-n}\left\|v ; L_{2, r}^{r}(\Omega ; \sigma, \vec{g})\right\|^{2}
\end{gathered}
$$

We proceed to estimating the third integral in the left hand side of inequality (3.15). Applying inequalities (2.1), we have

$$
\begin{aligned}
& \int_{\Omega} \sigma^{2}(y)\left(g_{1}(y) g_{2}(y) \ldots g_{n}(y)\right)^{-2 r-1} \varepsilon^{-n}\left(\int_{\Pi_{\varepsilon, \vec{g}}(y)}|v(z)|^{2} d z\right) d y \\
& \leqslant \varepsilon^{-n} \nu^{2}(\varepsilon) \lambda^{-2 r n-n}(\varepsilon) \int_{\Omega} \sigma^{2}(z)\left(g_{1}(z) g_{2}(z) \ldots g_{n}(z)\right)^{-2 r-1}|v(z)|^{2}\left(\int_{\Omega} \chi_{\varepsilon, \vec{g}}(z ; y) d y\right) d z .
\end{aligned}
$$

To estimate the internal integral, we employ Lemma 2.1 and arrive at the following inequality

$$
\begin{aligned}
& \int_{\Omega} \sigma^{2}(y)\left(g_{1}(y) g_{2}(y) \ldots g_{n}(y)\right)^{-2 r-1} \varepsilon^{-n}\left(\int_{\Pi_{\varepsilon, \vec{g}}(y)}|v(z)|^{2} d z\right) d y \\
& \quad \leqslant \nu^{2}(\varepsilon) \lambda^{-2 r n}(\varepsilon) 2^{-n}\left\|v ; L_{2, r}^{0}(\Omega ; \sigma, \vec{g})\right\|^{2} .
\end{aligned}
$$

By (3.15) it follows from the obtained inequalities (3.17) - 3.19) that

$$
\left(1-\widetilde{c}_{m}(\varepsilon)\right)\left\|v ; L_{2, r}^{r}(\Omega ; \sigma, \vec{g})\right\|^{2}-\widetilde{\mathbb{C}}_{m}(\varepsilon) \varepsilon^{-2 r}\left\|v ; L_{2, r}^{0}(\Omega ; \sigma, \vec{g})\right\|^{2} \leqslant \varepsilon^{-n} M_{m}(\varepsilon) \operatorname{Re} B_{\varepsilon, m}[v, v],
$$

where sesquilinear form $B_{\varepsilon, m}[v, v]$ is defined by identity (3.16) and

$$
\begin{aligned}
& \widetilde{c}_{m}=c_{m} \nu^{4}\left(\varepsilon_{m}\right) \lambda^{2 n}\left(\varepsilon_{m}\right)\left(1+\frac{1}{m}\right)^{n}, \varepsilon_{m}=\frac{\varepsilon m}{m+1}, \\
& \widetilde{\mathbb{C}}_{m}(\varepsilon)=\mathbb{C}_{m} \nu^{4}(\varepsilon) \lambda^{-2 r n}(\varepsilon) \lambda^{2 n}\left(\varepsilon_{m}\right)\left(1+\frac{1}{m}\right)^{n}, \\
& M_{m}(\varepsilon)=M_{0} \nu^{2}\left(\varepsilon_{m}\right) \lambda^{2 n}\left(\varepsilon_{m}\right) 2^{n}\left(1+\frac{1}{m}\right)^{n} .
\end{aligned}
$$

We introduce a new sesquilinear form

$B_{\varepsilon, m}^{(1)}[u, v]=\sum_{|k|=|l|=r} \int_{\Omega}\left(\int_{\Omega} \chi_{\varepsilon}^{(m)}(z ; y) a_{k l}(y) d y\right)\left(g_{1}(z) g_{2}(z) \ldots g_{n}(z)\right)^{-1} p_{k}(z) p_{l}(z) u^{(k)}(z) \overline{v^{(l)}(z)} d z$,

where functions $p_{k}(z)$ are defined by identity 3.2 . 
In view of the boundedness of coefficients $a_{k l}(x)$ as $|k|=|l|=r$, we have

$$
\begin{array}{r}
\left|B_{\varepsilon, m}^{(1)}[v, v]-B_{\varepsilon, m}[v, v]\right| \leqslant M \sum_{|k|=|l|=r} \int_{\Omega}\left(\int_{\Omega} \chi_{\varepsilon}^{(m)}(z ; y)\left|1-\frac{p_{k}(y) p_{l}(y)}{p_{k}(z) p_{l}(z)} \cdot \frac{g_{1}(z) g_{2}(z) \ldots g_{n}(z)}{g_{1}(y) g_{2}(y) \ldots g_{n}(y)}\right|\right. \\
\left.p_{k}(z) p_{l}(z)\left(g_{1}(z) g_{2}(z) \ldots g_{n}(z)\right)^{-1}\left|v^{(k)}(z)\right|\left|v^{l}(z)\right| d z\right) d y .
\end{array}
$$

By condition (2.1), for sufficiently small $\varepsilon>0$ there exists a positive number $\mu_{1}(\varepsilon)$ such that

$$
\left|1-\frac{p_{k}(y) p_{l}(y)}{p_{k}(z) p_{l}(z)} \cdot \frac{g_{1}(z) g_{2}(z) \ldots g_{n}(z)}{g_{1}(y) g_{2}(y) \ldots g_{n}(y)}\right| \leqslant \mu_{1}(\varepsilon)
$$

for all $y, z \in \Omega$ satisfying the condition $\chi_{\varepsilon, \vec{g}}^{(m)}(z ; y) \neq 0 ; \mu_{1}(\varepsilon) \rightarrow 0$ as $\varepsilon \rightarrow 0$. In view of this fact and by Lemma 2.1 and Cauchy-Schwarz inequality, it follows from (3.22) that

$$
\left|B_{\varepsilon, m}^{(1)}[v, v]-B_{\varepsilon, m}[v, v]\right| \leqslant \varepsilon^{n} \mu_{1}(\varepsilon)\left\|v ; L_{2, r}^{r}(\Omega ; \sigma, \vec{g})\right\|^{2}
$$

for all $v \in C_{0}^{\infty}(\Omega)$.

We consider a new sesquilinear form

$$
\begin{aligned}
& B_{\varepsilon, m}^{(2)}[u, v]=\sum_{|k|=|l|=r} \int_{\Omega}\left(\int_{\Omega} \chi_{\varepsilon, \vec{g}}^{(m)}(z ; y) d y\right) a_{k l}(z) p_{k}(z) p_{l}(z) \\
& \cdot\left(g_{1}(z) g_{2}(z) \ldots g_{n}(z)\right)^{-1} u^{(k)}(z) \overline{v^{(l)}(z)} d z, \quad\left(u, v \in C_{0}^{\infty}(\Omega)\right) .
\end{aligned}
$$

By condition (3.6), Lemma 2.1, arguing in a standard way by Cauchy-Schwarz inequality, we prove for each sufficiently small positive $\nu$ there exists number $\varepsilon_{\nu}>0$ such that

$$
\left|B_{\varepsilon, m}^{(1)}[v, v]-B_{\varepsilon, m}^{(2)}[v, v]\right| \leqslant \varepsilon^{n} \nu\left\|v ; L_{2, r}^{r}(\Omega ; \sigma, \vec{g})\right\|^{2}
$$

for all $v \in C_{0}^{\infty}(\Omega)$ and each $\varepsilon \in\left(0, \varepsilon_{\nu}\right)$.

Lemma 3.1. For each real-valued function $\Phi(z) \in L_{1}(\Omega)$ and for sufficiently small $\varepsilon>0$ the inequality

$$
\begin{aligned}
c_{n, m} \int_{\Omega} & \left(\int_{\Omega} \chi_{\varepsilon, \vec{g}}^{(m)}(z ; y) d y\right)\left(g_{1}(z) g_{2}(z) \ldots g_{n}(z)\right)^{-1} \Phi(z) d z \\
& \leqslant \lambda^{n}\left(\varepsilon_{m}\right) \varepsilon^{n} \int_{\Omega} \Phi(z) d z+\varepsilon^{n}\left[\lambda^{n}\left(\varepsilon_{m}\right)-\lambda^{-n}\left(\varepsilon_{m}\right)\right] \int_{\Omega} \Phi^{-}(z) d z
\end{aligned}
$$

holds true, where

$$
\Phi^{-}(z)=(|\Phi(z)|-\Phi(z)) / 2, c_{n, m}=2^{n}\left(1+\frac{1}{m}\right)^{n} .
$$

Proof. We introduce a function $\Phi^{+}(z)=(|\Phi(z)|+\Phi(z)) / 2$. We observe that $\Phi(z)=\Phi^{+}(z)-$ $\Phi^{-}(z)$ and functions $\Phi^{+}(z), \Phi^{-}(z)$ are non-negative.

By Lemma 2.1 we have

$$
\begin{aligned}
& \varepsilon^{n} 2^{-n} \lambda^{-n}\left(\varepsilon_{m}\right)\left(1+\frac{1}{m}\right)^{-n} \leqslant\left(g_{1}(z) g_{2}(z) \ldots g_{n}(z)\right)^{-1} \int_{\Omega} \chi_{\varepsilon, \vec{g}}^{(m)}(z ; y) d y \\
& \left(g_{1}(z) g_{2}(z) \ldots g_{n}(z)\right)^{-1} \int_{\Omega} \chi_{\varepsilon, \vec{g}}^{(m)}(z ; y) d y \leqslant \varepsilon^{n}\left(1+\frac{1}{m}\right)^{-n} 2^{-n} \lambda^{n}\left(\varepsilon_{m}\right) .
\end{aligned}
$$

We multiply both sides of the former inequality by $\Phi^{-}(z)$, while the latter is multiplied by $\Phi^{+}(z)$. Then we integrate in $z \in \Omega$. The obtained inequalities and the identity $\Phi(z)=\Phi^{+}(z)-\Phi^{-}(z)$ yield (3.26). 
Applying inequality 3.26 as

$$
\Phi(z)=\operatorname{Re} \sum_{|k|=|l|=r} a_{k l}(z) p_{k}(z) p_{l}(z) v^{(k)}(z) \overline{v^{(l)}(z)},
$$

we have

$$
c_{n, m} \operatorname{Re} B_{\varepsilon, m}^{(2)}[v, v] \leqslant \lambda^{n}\left(\varepsilon_{m}\right) \varepsilon^{n} \operatorname{Re} B[v, v]+\varepsilon^{n}\left[\lambda^{n}\left(\varepsilon_{m}\right)-\lambda^{-n}\left(\varepsilon_{m}\right)\right] \int_{\Omega} \Phi^{-}(z) d z .
$$

Since coefficients $a_{k l}(z),|k|=|l|=r$, are bounded, we have

$$
\begin{aligned}
\int_{\Omega} \Phi^{-}(z) d z \leqslant \int_{\Omega}|\Phi(z)| d z & \leqslant M \sum_{|k|=|l|=r} \int_{\Omega} p_{k}(z) p_{l}(z)\left|v^{(k)}(z) \| v^{(l)}(z)\right| d z \\
& \leqslant M\left\|v ; L_{2, r}^{r}(\Omega ; \sigma, \vec{g})\right\|^{2} .
\end{aligned}
$$

Together with (3.27) this implies that

$$
\operatorname{Re} B_{\varepsilon, m}^{(2)}[v, v] \leqslant \varepsilon^{n} M\left\{\operatorname{Re} B[v, v]+\mu_{2}(\varepsilon)\left\|v ; L_{2, r}^{r}(\Omega ; \sigma, \vec{g})\right\|^{2}\right\},
$$

where $M$ is a positive number independent of $\varepsilon>0$ and $v(x)$, and

$$
\mu_{2}(\varepsilon)=\lambda^{n}\left(\varepsilon_{m}\right)-\lambda^{-n}\left(\varepsilon_{m}\right), \quad \varepsilon_{m}=\frac{\varepsilon m}{m+1} .
$$

Employing inequality (3.20), we have

$$
\begin{aligned}
\left(1-\widetilde{c}_{m}(\varepsilon)\right)\left\|v ; L_{2, r}^{r}(\Omega ; \sigma, \vec{g})\right\|^{2} & -\widetilde{\mathbb{C}}_{m}(\varepsilon) \varepsilon^{-2 r}\left\|v ; L_{2, r}^{0}(\Omega ; \sigma, \vec{g})\right\|^{2} \\
\leqslant & \varepsilon^{-n} M_{m}(\varepsilon) \operatorname{Re} B_{\varepsilon, m}^{(2)}[v, v]+\varepsilon^{-n} M_{m}(\varepsilon)\left|B_{\varepsilon, m}[v, v]-B_{\varepsilon, m}^{(1)}[v, v]\right| \\
& +\varepsilon^{-n} M_{m}(\varepsilon)\left|B_{\varepsilon, m}^{(1)}[v, v]-B_{\varepsilon, m}^{(2)}[v, v]\right| .
\end{aligned}
$$

By inequalities (3.24), (3.25) it follows that

$$
\begin{aligned}
\left(1-\widetilde{c}_{m}(\varepsilon)\right)\left\|v ; L_{2, r}^{r}(\Omega ; \sigma, \vec{g})\right\|^{2} & -\widetilde{\mathbb{C}}_{m}(\varepsilon) \varepsilon^{-2 r}\left\|v ; L_{2, r}^{0}(\Omega ; \sigma, \vec{g})\right\|^{2} \\
& \leqslant \varepsilon^{-n} M_{m}(\varepsilon) \operatorname{Re} B_{\varepsilon, m}^{(2)}[v, v]+\mu_{1}(\varepsilon) M_{m}(\varepsilon)\left\|v ; L_{2, r}^{r}(\Omega ; \sigma, \vec{g})\right\|^{2} \\
& +M_{m}(\varepsilon) \nu\left\|v ; L_{2, r}^{r}(\Omega ; \sigma, \vec{g})\right\|^{2} .
\end{aligned}
$$

Applying inequality (3.28), we get

$$
\begin{aligned}
\left(1-\widetilde{c}_{m}(\varepsilon)\right)\left\|v ; L_{2, r}^{r}(\Omega ; \sigma, \vec{g})\right\|^{2}- & \widetilde{\mathbb{C}}_{m}(\varepsilon) \varepsilon^{-2 r}\left\|v ; L_{2, r}^{0}(\Omega ; \sigma, \vec{g})\right\|^{2} \\
\leqslant & M_{m}(\varepsilon) M\left\{\operatorname{Re} B[v, v]+\mu_{2}(\varepsilon)\left\|v ; L_{2, r}^{r}(\Omega ; \sigma, \vec{g})\right\|^{2}\right\} \\
& +\mu_{1}(\varepsilon) M_{m}(\varepsilon)\left\|v ; L_{2, r}^{r}(\Omega ; \sigma, \vec{g})\right\|^{2}+M_{m}(\varepsilon) \nu\left\|v ; L_{2, r}^{r}(\Omega ; \sigma, \vec{g})\right\|^{2} .
\end{aligned}
$$

This yields that

$$
\begin{aligned}
{\left[1-\widetilde{c}_{m}(\varepsilon)-\mu_{2}(\varepsilon) M_{m}(\varepsilon) M\right.} & \left.-\mu_{1}(\varepsilon) M_{m}(\varepsilon)-M_{m}(\varepsilon) \nu\right]\left\|v ; L_{2, r}^{r}(\Omega ; \sigma, \vec{g})\right\|^{2} \\
& -\widetilde{\mathbb{C}}_{m}(\varepsilon) \varepsilon^{-2 r}\left\|v ; L_{2, r}^{0}(\Omega ; \sigma, \vec{g})\right\|^{2} \leqslant M_{m}(\varepsilon) M \operatorname{Re} B[v, v],
\end{aligned}
$$

where numbers $\widetilde{c}_{m}(\varepsilon), \widetilde{\mathbb{C}}_{m}(\varepsilon), M_{m}(\varepsilon)$ are the same as in 3.21$), \mu_{1}(\varepsilon)$ is the same as in (3.23), while number $\mu_{2}(\varepsilon)$ is determined by identity (3.29).

Choosing number $m$ large enough and numbers $\varepsilon, \nu$ small enough, by 3.30 we obtain the inequality

$$
\operatorname{Re} B[v, v] \geqslant c_{3}\left\|v ; L_{2, r}^{r}(\Omega ; \sigma, \vec{g})\right\|^{2}-c_{4}\left\|v ; L_{2, r}^{0}(\Omega ; \sigma, \vec{g})\right\|^{2}
$$

for all $v \in C_{0}^{\infty}(\Omega) ; c_{3}, c_{4}$ are positive constants independent of $v(x)$. 
Thus, we have completed the proof of the Gårding inequality for the degenerate elliptic operator associated with sesquilinear form $B[u, v]$ defined by identity $(3.3)$ in the case $a_{k l} \equiv 0$ as $|k|+|l| \leqslant 2 r-1$.

We proceed to the proof of Gårding inequality (3.7) in the general case. We let

$$
B_{0}[u, v]=\sum_{|k|=|l|=r} \int_{\Omega} p_{k}(x) p_{l}(x) a_{k l}(x) u^{(k)}(x) \overline{v^{(l)}(x)} d x, \quad B_{1}[u, v]=B[u, v]-B_{0}[u, v] .
$$

In accordance with the above proven result for sesquilinear form $B_{0}[u, v]$ we have inequality (3.31), i.e., there exist numbers $c_{5}>0, c_{6} \geqslant 0$ such that

$$
\operatorname{Re} B_{0}[u, u] \geqslant c_{5}\left\|u ; L_{2, r}^{r}(\Omega ; \sigma, \vec{g})\right\|^{2}-c_{6}\left\|u ; L_{2, r}^{0}(\Omega ; \sigma, \vec{g})\right\|^{2}
$$

for all $u \in C_{0}^{\infty}(\Omega)$.

We represent sesquilinear form $B_{1}[u, v]$ as

$$
B_{1}[u, v]=B_{11}[u, v]+B_{12}[u, v],
$$

where

$$
\begin{aligned}
& B_{11}[u, v]=\sum_{|k|<r,|l| \leqslant r} \int_{\Omega} p_{k}(x) p_{l}(x) a_{k l}(x) u^{(k)}(x) \overline{v^{(l)}(x)} d x, \\
& B_{12}[u, v]=\sum_{|k|=r,|l|<r} \int_{\Omega} p_{k}(x) p_{l}(x) a_{k l}(x) u^{(k)}(x) \overline{v^{(l)}(x)} d x .
\end{aligned}
$$

Employing Hölder inequality with exponents $q_{k l}, \lambda_{k l}$, by the condition

$$
\left\|a_{k l} ; L_{q_{k l}}\left(\Omega ;\left(g_{1} g_{2} \ldots g_{n}\right)^{-1 / q_{k l}}\right)\right\|<+\infty,|k|+|l| \leqslant 2 r-1,
$$

we have

$$
\begin{aligned}
\mid B_{11}[u, v] & \leqslant \sum_{|k|<r,|l| \leqslant r} \int_{\Omega}\left(g_{1}(x) g_{2}(x) \ldots g_{n}(x)\right)^{-1 / q_{k l}}\left|a_{k l}(x)\right| \sigma_{k l}(x)\left|u^{(k)}(x) \| v^{(l)}(x)\right| d x \\
& \leqslant M_{0} \sum_{|k|<r,|l| \leqslant r}\left\|u^{(k)} v^{(l)} ; L_{\lambda_{k l}}\left(\Omega ; \sigma_{k l}\right)\right\| .
\end{aligned}
$$

Applying then Lemma 2.4 as $p=q=2$, we obtain

$$
\mid B_{11}[u, v] \leqslant M\left\|v ; W_{2}^{r}(\Omega ; \sigma, \vec{g})\right\|\left\{\tau\left\|u ; W_{2}^{r}(\Omega ; \sigma, \vec{g})\right\|+c_{0} \tau^{-\mu}\left\|u ; L_{2, r}^{0}(\Omega ; \sigma, \vec{g})\right\|\right\},
$$

where $\mu=\max _{|k|<r} \mu_{k}$ and numbers $\mu_{k}$ are determined by identity 2.14); $\tau$ is a sufficiently small positive number.

Since sesquilinear form $B_{12}[u, v]$ involves $u^{(k)}(x) v^{(l)}(x) \quad|l|<r,|k|=r$, (see (3.34)), by switching the roles of $u(x)$ and $v(x)$ and proceeding as in the proof of inequality (3.35), we have

$$
\mid B_{12}[u, v] \leqslant M\left\|u ; W_{2}^{r}(\Omega ; \sigma, \vec{g})\right\|\left\{\tau\left\|v ; W_{2}^{r}(\Omega ; \sigma, \vec{g})\right\|+c_{0} \tau^{-\mu}\left\|v ; L_{2, r}^{0}(\Omega ; \sigma, \vec{g})\right\|\right\} .
$$

Employing inequalities (3.35), 3.36) as $u(x) \equiv v(x)$ and identity (3.33), we obtain

$$
\mid B_{1}[u, u] \leqslant M\left\|u ; W_{2}^{r}(\Omega ; \sigma, \vec{g})\right\|\left\{\tau\left\|u ; W_{2}^{r}(\Omega ; \sigma, \vec{g})\right\|+c_{0} \tau^{-\mu}\left\|u ; L_{2, r}^{0}(\Omega ; \sigma, \vec{g})\right\|\right\} .
$$

Since $B[u, v]=B_{0}[u, v]+B_{1}[u, v]$, by employing inequalities 3.32), 3.37) we find

$$
\begin{aligned}
\operatorname{Re} B[u, u] & \geqslant \operatorname{Re} B_{0}[u, u]-\left|B_{1}[u, u]\right| \\
& \geqslant\left(c_{5}-\tau\right)\left\|u ; L_{2, r}^{r}(\Omega ; \sigma, \vec{g})\right\|^{2}-\left(\tau+c_{0} \tau^{-2 \mu}+c_{6}\right)\left\|u ; L_{2, r}^{0}(\Omega ; \sigma, \vec{g})\right\|^{2} .
\end{aligned}
$$

Fixing a sufficiently small number $\tau>0$, we obtain

$$
\operatorname{Re} B[u, u] \geqslant c_{7}\left\|u ; L_{2, r}^{r}(\Omega ; \sigma, \vec{g})\right\|^{2}-c_{8}\left\|u ; L_{2, r}^{0}(\Omega ; \sigma, \vec{g})\right\|^{2}
$$


Since

$$
\left\|u ; W_{2}^{r}(\Omega ; \sigma, \vec{g})\right\|=\left\{\left\|u ; L_{2, r}^{r}(\Omega ; \sigma, \vec{g})\right\|^{2}+\left\|u ; L_{2, r}^{0}(\Omega ; \sigma, \vec{g})\right\|^{2}\right\}^{1 / 2},
$$

obtained inequality (3.38) implies inequality (3.7). The proof of Theorem 3.1 is complete.

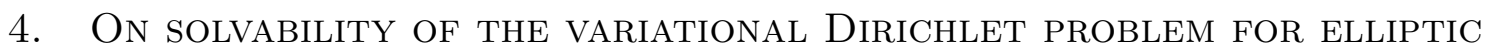 OPERATORS WITH NON-POWER DEGENERATION}

In this section we formulate a result on solvability of the, which can be proved by means of the above obtained Gårding inequality and a generalized Lax-Milgram theorem (see, for instance, [8]).

Let $\left(W_{2}^{r}(\Omega ; \sigma, \vec{g})\right)^{\prime}$ be a space of bounded antilinear continuous functionals defined on $W_{2}^{r}(\Omega ; \sigma, \vec{g})$ and equipped with the norm of the dual space.

We consider the following Dirichlet variational problem associated with sesquilinear form (3.3).

Problem $D_{\lambda}$. Given a functional $F \in\left(W_{2}^{r}(\Omega ; \sigma, \vec{g})\right)^{\prime}$, find a solution $U(x)$ to the equation

$$
B[U, v]+\int_{\Omega} \sigma^{2}(x)\left(g_{1}(x) g_{2}(x) \cdots g_{n}(x)\right)^{-2 r} U(x) \overline{v(x)} d x=\langle F, v\rangle, v \in C_{0}^{\infty}(\Omega)
$$

in the space $W_{2}^{r}(\Omega ; \sigma, \vec{g})$.

We observe that if coefficients $a_{k l}(x)$ and right hand side $F$ of equation (4.1) are smooth enough, solution $U(x)$ of Problem $D_{\lambda}$ satisfies the differential equation

$$
(A U)(x)+\lambda \sigma^{2}(x)\left(g_{1}(x) g_{2}(x) \cdots g_{n}(x)\right)^{-2 r} U(x)=F(x), \quad x \in \Omega,
$$

where $A$ is the same differential operator as in (3.1).

Theorem 4.1. Assume the hypothesis of Theorem 3.1. Then there exists a number $\lambda_{0} \geqslant 0$ such that as $\lambda>\lambda_{0}$, for each given functional $F \in\left(W_{2}^{r}(\Omega ; \sigma, \vec{g})\right)^{\prime}$ Problem $D_{\lambda}$ has the unique solution $U(x)$ and the estimate

$$
\left\|U ; W_{2}^{r}(\Omega ; \sigma, \vec{g})\right\| \leqslant M\left\|F ;\left(W_{2}^{r}(\Omega ; \sigma, \vec{g})\right)^{\prime}\right\|
$$

is satisfied, where positive number $M$ is independent of $F$.

\section{On solvability of Dirichlet VARiational PROBlem With inhomogeneous BOUNDARY CONDITIONS IN A HALP-SPACE}

Since class $C_{0}^{\infty}(\Omega)$ is dense in $W_{2}^{r}(\Omega ; \sigma, \vec{g})$, Theorem 2 allows us to establish the solvability of the variational Dirichlet problem with homogeneous boundary conditions for particular types of domains $\Omega \subset R_{n}$ (for instance, bounded domains, complements to bounded domains, half-space), while for studying the solvability of such problems with inhomogeneous boundary conditions we need first to prove embedding theorem of various metrics for appropriate weighted spaces, whose elements, in the general situation, are not approximated by the functions in $C_{0}^{\infty}(\Omega)$. In this section we formulate the result (without proof) on the solvability of the Dirichlet variational problem for one class of elliptic problems in the half-space with a power degeneration.

Let $R_{n}^{+}=\left\{x \mid x=\left(x^{\prime}, x_{n}\right) \in R_{n}, x_{n}>0\right\}$ and let function $\varphi(t) \in C^{\infty}\left(R_{1}^{+}\right)$be so that $0 \leqslant \varphi(t) \leqslant 1$ for each $t \in\left[\frac{1}{2} ; 1\right]$ and $\varphi(t) \equiv 0$, as $t \geqslant 1 ; \varphi(t)=1$ for each $t \in\left[0 ; \frac{1}{2}\right]$. For all real numbers $\alpha, \beta$ we introduce the function

$$
\sigma_{\alpha, \beta}(t)=\varphi(t) t^{-\alpha}+(1-\varphi(t)) t^{\beta} \quad(t>0) .
$$

We denote by $W_{p ; \alpha, \beta, \gamma}^{r}\left(R_{n}^{+}\right)$the space of functions $u(x)\left(x \in R_{n}^{+}\right)$with a finite norm

$$
\left\|u ; W_{p ; \alpha, \beta, \gamma}^{r}\left(R_{n}^{+}\right)\right\|=\left\{\left\|u ; L_{p ; \alpha, \beta}^{r}\left(R_{n}^{+}\right)\right\|^{p}+\left\|u ; L_{p ; \alpha, \gamma}^{0}\left(R_{n}^{+}\right)\right\|^{p}\right\}^{1 / p},
$$


where

$$
\left\|u ; L_{p ; \alpha, \beta}^{r}\left(R_{n}^{+}\right)\right\|=\left\{\sum_{|k|=r} \int_{R_{n}^{+}}\left(\sigma_{\alpha, \beta}\left(x_{n}\right)\left|u^{(k)}(x)\right|\right)^{p} d x\right\}^{1 / p} .
$$

Let $\widetilde{C}_{0}^{\infty}\left(R_{n}^{+}\right)$be a set of infinitely differentiable in $R_{n}^{+}$functions compactly supported above, i.e., vanishing for large $x_{n}$. The symbols $\stackrel{\circ}{W}_{p ; \alpha, \beta, \gamma}^{r}\left(R_{n}^{+}\right), \widetilde{W}_{p ; \alpha, \beta, \gamma}^{r}\left(R_{n}^{+}\right)$stand for the closures of classes $C_{0}^{\infty}\left(R_{n}^{+}\right)$and $\widetilde{C}_{0}^{\infty}\left(R_{n}^{+}\right)$in space $W_{p ; \alpha, \beta, \gamma}^{r}\left(R_{n}^{+}\right)$, respectively.

We consider the sesquilinear form

$$
\mathbb{B}[u, v]=\sum_{|k|,|l| \leqslant r} \int_{R_{n}^{+}} \sigma_{\alpha, \beta}^{2}\left(x_{n}\right) a_{k l}(x) u^{(k)}(x) \overline{v^{(l)}(x)} d x
$$

and the associated Dirichlet variational problem.

Problem D. Given a functional $F \in\left(\stackrel{\circ}{W}_{2 ; \alpha, \beta, \gamma}^{r}\left(R_{n}^{+}\right)\right)^{\prime}$ and an element $\Psi(x) \in \widetilde{W}_{2 ; \alpha, \beta, \gamma}^{r}\left(R_{n}^{+}\right)$, find a solution $U(x) \in \widetilde{W}_{2 ; \alpha, \beta, \gamma}^{r}\left(R_{n}^{+}\right)$to the equation

$$
\mathbb{B}[U, v]=<F, v>, \forall v \in C_{0}^{\infty}\left(R_{n}^{+}\right),
$$

satisfying the condition

$$
U(x)-\Psi(x) \in \stackrel{\circ}{W}_{2 ; \alpha, \beta, \gamma}^{r}\left(R_{n}^{+}\right) .
$$

Assume that coefficients $a_{k l}(x)$ of sesquilinear form (5.1) obey the conditions:

I) as $|k|=|l|=r$, coefficients $a_{k l}(x)$ are bounded and satisfy the ellipticity condition:

$$
\operatorname{Re} \sum_{|k|=|l|=r} a_{k l}(x) \xi^{k} \xi^{l} \geqslant c|\xi|^{2 r}
$$

for all $x \in R_{n}^{+}, \xi \in R_{n}$, where $c$ is a positive number independent of $x, \xi$, and for all sufficiently small $\nu>0$ there exists a number $\varepsilon>0$ such that

$$
\left|a_{k l}(x)-a_{k l}(y)\right|<\nu
$$

for all $x, y \in R_{n}^{+}$such that

$$
\left|x_{i}-y_{i}\right|<\frac{1}{2} \varepsilon y_{n}, i=\overline{1, n}
$$

II) as $|k|+|l| \leqslant 2 r-1$, coefficients $a_{k l}$ belong to space $L_{p_{k l}}\left(R_{n}^{+} ; \sigma_{\alpha_{k l}, \beta_{k l}}\left(x_{n}\right)\right)$, where numbers $\alpha_{k l}, \beta_{k l}, p_{k l}$ are determined by the relations:

a) if $|k|<r,|l|<r$, then

$$
\begin{aligned}
\alpha_{k l} & =-\frac{n}{2}+\frac{n}{p_{k l}}+n \delta_{k}-r+|l|, \\
\beta_{k l} & =2 r-|k|-|l|-(n-1) \delta_{k}+\frac{n}{2}-\frac{n}{p_{k l}}, \\
\frac{1}{p_{k l}} & =1-\delta_{k}-\varepsilon_{l}-\left(\frac{1}{2}-\frac{r-|l|}{n}\right)_{+},
\end{aligned}
$$

where numbers $\delta_{k}, \varepsilon_{l}$ in the interval $(0,1 / 2)$ satisfy the conditions

$$
0<\frac{1}{2}-\delta_{k}-\varepsilon_{l}<\min \left\{\frac{1}{2}, \frac{r-|k|}{n}\right\}
$$

and $(\theta)_{+}=\theta$ if $\theta>0$ and $(\theta)_{+}=0$ otherwise;

b) if $|k|=r,|l| \leqslant r-1$, then

$$
\alpha_{k l}=\frac{n}{p_{k l}}-r+|l|
$$




$$
\begin{aligned}
& \beta_{k l}=-\frac{n}{p_{k l}}+r-|l|, \\
& \frac{1}{p_{k l}}=\frac{1}{2}-\varepsilon_{0}-\left(\frac{1}{2}-\frac{r-|l|}{n}\right)_{+},
\end{aligned}
$$

and $\varepsilon_{0}$ is a sufficiently small positive number;

c) if $|k| \leqslant r-1|l|=r$, then

$$
\begin{aligned}
\alpha_{k l} & =\frac{1}{2}-\delta_{k}, \\
\beta_{k l} & =r-|k|-\frac{1}{2}+\delta_{k}, \\
\frac{1}{p_{k l}} & =\frac{1}{2}-\delta_{k} .
\end{aligned}
$$

In these conditions number $\delta_{k}$ is such that

$$
\frac{1}{2}-\frac{1}{n} \leqslant \delta_{k}<\frac{1}{2}
$$

Theorem 5.1. Assume that conditions I), II) are satisfied and that there exists a positive number $c_{0}$ such that

$$
c_{0} \int_{R_{n}^{+}}\left(\sigma_{\alpha, \beta}\left(x_{n}\right) x_{n}^{-r}|v(x)|\right)^{2} d x \leqslant \operatorname{Re} \mathbb{B}[v, v]
$$

for all $v \in C_{0}^{\infty}\left(R_{n}^{+}\right)$. Suppose also that

$$
\alpha<-\frac{1}{2},-\alpha+\frac{1}{2} \notin\{1,2, \ldots, r\}, \beta+\frac{1}{2} \notin\{1,2, \ldots, r\}, \beta-r \geqslant \gamma .
$$

Then for each given functional $F \in\left(\stackrel{\circ}{W}_{2 ; \alpha, \beta, \gamma}^{r}\left(R_{n}^{+}\right)\right)^{\prime}$ and each given element $\Psi \in \widetilde{W}_{2 ; \alpha, \beta, \gamma}^{r}\left(R_{n}^{+}\right)$ Problem $D$ has the unique solution $U(x)$ and the estimate

$$
\left\|U ; W_{2 ; \alpha, \beta, \gamma}^{r}\left(R_{n}^{+}\right)\right\| \leqslant M\left\{\left\|F ;\left(\stackrel{\circ}{W}_{2 ; \alpha, \beta, \gamma}^{r}\left(R_{n}^{+}\right)\right)^{\prime}\right\|+\left\|\Psi ; W_{2 ; \alpha, \beta, \gamma}^{r}\left(R_{n}^{+}\right)\right\|\right\}
$$

holds true, where number $M$ is independent of $F$ and $\Psi$.

Remark 5.1. Condition (5.2) means that on the hyperplane $x_{n}=0$ the solution $U(x)$ to Problem $D$ has the same traces as given function $\Psi(x)$. Under additional restrictions for parameters $\alpha, \beta, \gamma$, as in work [9], one can write explicitly the boundary conditions on the hyperplane $x_{n}=0$.

\section{BIBLIOGRAPHY}

1. K. Yosida. Functional analysis. Springer-Verlag, Berlin (1965).

2. Yu.V. Egorov. Lectures on partial differential equations. Supplementary chapters. Izd-vo MGU, Moscow (1985). (in Russian).

3. L. Gårding. Dirichlet's problem for linear elliptic partial differential equations // Math. Scand. 1:1, 55-72 (1953).

4. L. D. Kudryavtsev. Direct and inverse imbedding theorems. Applications to the solution of elliptic equations by variational methods // Trudy Mat. Inst. Steklov. Acad. Sci. USSR. 55, 3-182 (1959). (in Russian).

5. P.I. Lizorkin, S.M. Nikol'skii. Coercive properties of elliptic equations with degeneration. Variational method // Trudy Mat. Inst. Steklov. 157, 90-118 (1981). (in Russian).

6. P.I. Lizorkin, S.M. Nikol'skii. Coercive properties of an elliptic equation with degeneration and a generalized right-hand side // Trudy Mat. Inst. Steklov. 161, 157-183 (1983). [Proc. Steklov Inst. Math. 161, 171-198 (1984).] 
7. N.V. Miroshin. Dirichlet variational problem for an elliptic operator singular on the boundary // Differ. Uravn. 24:3, 455-464 (1988). [Diff. Equat. 24:3, 323-329 (1988).]

8. S.M. Nikol'skil, P.I. Lizorkin, N.V. Miroshin. Weighted function spaces and their applications to the investigation of boundary value problems for degenerate elliptic equations // Izv. Vyssh. Uchebn. Zaved. Mat. 8, 4-30 (1988). [Soviet Math. Izv. VUZ. Matem. 32:8, 1-40 (1988).]

9. S.A. Iskhokov. On the smoothness of the solution of degenerate differential equations // Differ. Uravn. 31:4, 641-653 (1995). [Diff. Equat. 31:4, 594-606 (1995).]

10. S.A. Iskhokov. Smoothness of the generalized solution of an elliptic equation with nonpower degeneracy // Differ. Uravn. 39:11, 1536-1542 (2003). [Diff. Equat. 39:11, 1618-1625 (2003).]

11. I.A. Kipriyanov. Garding's inequality for degenerate elliptic operators, and its applications // Trudy Mat. Inst. Steklov. 105, 77-88 (1969). [Proc. Steklov Inst. Math. 105, 92-104 (1969).]

12. S.A. Iskhokov. Gårding's inequality for elliptic operators with degeneracy // Matem. Zametki. 87:2, 201-216 (2010). [Math. Notes. 87:2, 189-203 (2010).]

13. S.A. Iskhokov, M.G. Gadoev, I.A. Yakushev. Garding's inequality for higher order elliptic operators with nonpower degeneration // Doklady RAN. 443:3, 286-289. [Dokl. Math. 85:2, 215-218 (2012).]

14. P.I. Lizorkin. Estimate of mixed and intermediate derivatives in weighted $L_{p}$-norms // Trudy Mat. Inst. Steklov. 156, 130-142 (1980). [Proc. Steklov Inst. Math. 156, 141-153 (1983).]

15. M. Troizi. Theoremi di inclusione negli spazi di Sobolev con peso // Ricerche Mat. 18, 49-74 (1969).

16. V.I. Burenkov. Sobolev Spaces on Domains. Teubner-Texte Math. 137. B.G.Teubner, Stuttgart. (1998).

Sulaimon Abunasrovich Iskhokov,

Institute of Mathematics named after A. Dzhuraev AS RT,

Aini str. 299/4,

734063, Dushanbe, Tadzhikistan,

Mirny Polytechnic Institute,

a branch of North-Eastern Federal University named after M.K. Ammosov,

Tikhonova str., 5/1,

678170, Mirny, Republic of Sakha (Yakutia), Russia

E-mail: sulaimon@mail.ru

Makhmadrakhim Gafurovich Gadoev,

Mirny Polytechnic Institute,

a branch of North-Eastern Federal University named after M.K. Ammosov,

Tikhonova str., 5/1,

678170, Mirny, Republic of Sakha (Yakutia), Russia

E-mail: gadoevorambler.ru

Ilya Anatolievich Yakushev,

Mirny Polytechnic Institute,

a branch of North-Eastern Federal University named after M.K. Ammosov,

Tikhonova str., 5/1,

678170, Mirny, Republic of Sakha (Yakutia), Russia

E-mail: yakushevilya@mail.ru 